\title{
Segmental rigidity and spinal myoclonus as a paraneoplastic syndrome
}

\author{
T H ROOBOL, $*$ B A KAZZAZ, $\dagger$ CH J VECHT $\ddagger$ \\ From the Department of Neurology, ${ }^{*} \ddagger$ Diaconessenhuis Voorburg, the Department of Pathology, $\dagger$ Leyenburg \\ Ziekenhuis The Hague, and the Comprehensive Cancer Center West, $\ddagger$ Leyden, The Netherlands
}

SUMMARY A 68 year old woman is described with persisting muscular rigidity of the left lower leg $\vec{\circ}$ together with transient myoclonic jerking in the left quadriceps muscle. Six weeks after onset a small $\overrightarrow{\vec{\omega}}$ cell carcinoma of the lung became manifest. With radiotherapy and chemotherapy completeo remission was achieved. Segmental muscular spasm improved at the same time. Necropsy revealed $\overline{3}$ loss and degeneration of alpha-motor neuron cells at one side of the anterior horn of the lumbar enlargement. This case may represent another manifestation of paraneoplastic subacute motoro neuronopathy.

Muscular rigidity due to spinal dysfunction and spinal myoclonus are thought to result from absent or disordered inhibition of alpha-motor neurons respectively. ${ }^{1-5}$ The presence of both these symptoms together has been reported in one case of encephalomyelitis. ${ }^{6}$ We describe another patient with symptoms of segmental rigidity and spinal myoclonus which were followed after 6 weeks by the appearance of a small cell carcinoma of the lung. Histology of the spinal cord offered an explanation for the clinical findings and indicated a paraneoplastic aetiology.

\section{Case report}

A 68 year old woman was admitted in July 1984 because of an intensely painful spasm of the left calf and foot. Six days earlier she had noticed involuntary jerking movements in the left knee and ankle. Gradually these had become more intense until the left foot was constantly held in plantar flexion. No paraesthesias were felt. Micturition was undisturbed. A month before she had complained of pain and itching in a circumscribed segmental zone on the left side of the chest. This had been interpreted as beginning shingles, but an eruption never occurred. Gradually the pain had subsided and only a "queer" sensation in the same region persisted. The further history was unremarkable. On

Address for reprint requests: Dr CJ Vecht, Comprehensive Cancer Centre West, Opaalstraat 5, 2332 TA Leyden, The Netherlands.

Received 30 April 1986.

Accepted 10 July 1986 examination an alert afebrile woman was seen who seemed exasperated by the pain in her leg. The left knee was held in flexion; stretching it exacerbated the pain in the calf. The eff foot was fixed in plantar flexion, the big toe in extension. active plantar or dorsal flexion of the foot or toes, was posit- $-\infty$ ble. On palpation both the soleus and tibialis anterior muscte. groups were contracting. Sometimes regular myoclonic movements at a rate of about one contraction per second in the ankle or knee were seen, especially with the legs hanging. No fasciculations were seen. No specific sensory stimuli exacerbating the contractions could be found. Muscle powero in the affected leg was difficult to assess; proximal musclee power was intact. Sensation was undisturbed except for $a \overrightarrow{\vec{\sigma}}$ well demarcated dysaesthetic area at the level of D8 on the $\frac{0}{3}$ left. The patellar reflex on the left was increased. The remainder of the neurological examination, including the mental status, was normal. General physical examination revealed no other abnormalities.

Laboratory studies gave normal results including WR, anti-nuclear antibody, circulating immune complexes ando angiotensin-converting enzyme. Cerebrospinal fluid showed 3 .

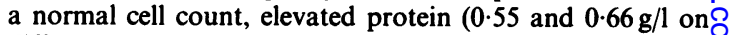
different occasions), elevated IgG index $(0.85,0.75$ and 2.073 on different occasions) with a small spike of gamma-globulin 0 on electrophoresis which was not seen in serum. Cytology was negative. A chest radiograph was normal. Muscle $?$ biopsy from the left anterior tibial muscle showed foci of muscle fibres with a variable transverse diameter and locally degenerating nuclei. Scattered areas of polynuclear-s inflammatory infiltrate with some necrosis and histiocytes $N$ were present. The findings were thought to be compatible with intense continued muscular activity. The electro- $\sigma$ myogram showed normal action potentials with continuous motor activity in the left tibialis anterior and triceps surae 

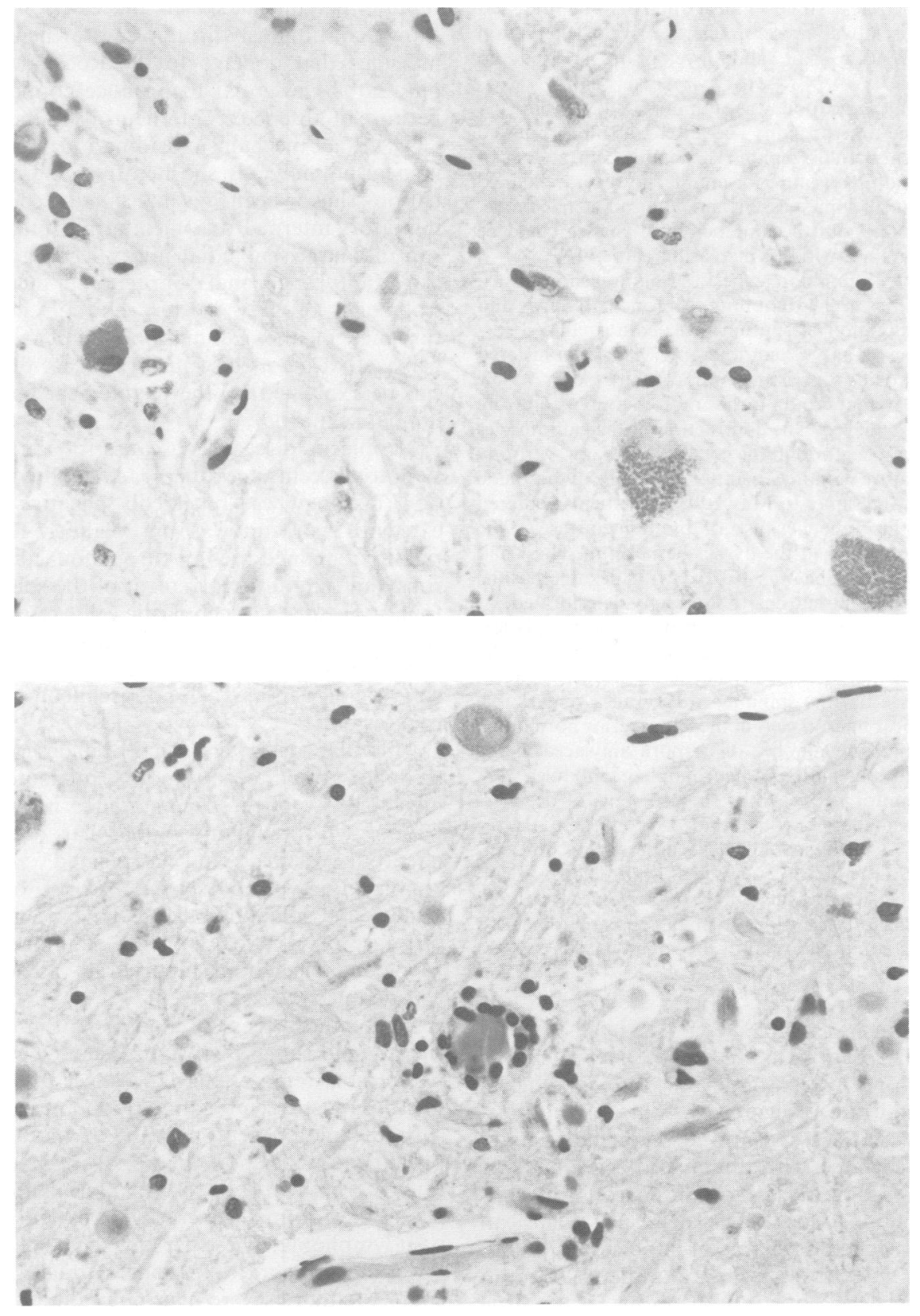

Fig 1 (a) Degeneration of ganglion cells with some bizarre forms. Increased number of glial cells with one macrophage can be seen. Tissue taken from anterior grey horn, lumbar segment. $(200 \times)$ (b) Neuronophagic nodule is present in association with several corpora amylacea. Increased number of glial cells. $(200 \times)$ 
muscles. Sometimes slight motor activity in the left quadriceps muscle was present. There was no denervation activity. EEG, CT scan and myelogram showed no abnormalities.

Prednisolone medication was administered without any effect on muscle tone or pain. The latter was much relieved during treatment with diazepam. The tetanic contractions of triceps surae and tibialis anterior muscle, however, remained almost unchanged; some passive and, later on, active movements in the ankle joint became possible. The leg could be stretched in the knee without increasing pain.

Six weeks after admission a chest radiograph showed suspect abnormalities in the right lung. A bronchial biopsy and cytologic investigation of sputum revealed a small cell carcinoma of the lung. Chemotherapy was administered (cyclophosphamide, vincristine, adriamycin). After a few cycles of treatment and local radiotherapy complete remission was achieved. The contraction of the calf muscles started to show relaxation in the course of therapy. Atrophy of calf musculature was prominent by now. The dysaesthetic zone remained the same. In May 1985 the patient reentered the hospital, this time because of brain metastases. The spasm in the left calf and the dysaesthesia on the chest had remained unaltered. She was treated with dexamethasone and radiotherapy, but her health gradually deteriorated.

At necropsy multiple metastases in liver, adrenal glands, pancreas, pleura, peritoneum and the brain were found. The spinal cord was macroscopically normal. On microscopical examination (see fig) the spinal cord showed a remarkable decrease of motor neuron cells in the left anterior horn in the lumbar swelling. The number of corpora amylacea in the grey matter was increased and neuronophagic nodules were present. The motor neuron cells showed signs of degeneration with smaller and rounded contours. Intracytoplasmatic granulovacuolar degeneration was present. Interneurons could not be recognised as such. There were no signs of demyelination. The spinal roots showed scattered areas of neoplastic infiltration of small cell carcinoma. No neoplastic cells could be found in the spinal cord.

\section{Discussion}

The muscular spasm restricted to the lower leg, the absence of long tract signs and the histological findings all indicate a segmental origin of the muscular rigidity. Muscle spasm and segmental rigidity have been associated with diminished cell counts or abnormal histology of alpha-motoneurons or complete isolation due to loss of interneurons. ${ }^{25}$ This can result in a continued discharge of impulses leading to muscle spasm. Electrophysiological evidence of spontaneous discharge of motoneurons was little influenced by reflex mechanisms or voluntary effort. ${ }^{3}$ We found similar findings in our case with a reduced number of small alpha-motoneurons which showed abnormal forms and loss of dendrites. It is important to note, however, that the presence or degeneration of interneurons was difficult to assess by light microscopy, because of the difficulties in differentiating them from microglia cells and astrocytes. ${ }^{3}$ In a case of con- tinuous hemifacial spasm the electromyogram has $\mathbb{D}$ shown continuously firing of motor neurons,${ }^{4}$ which indicates that damage to the Renshaw mechanism may not be essential to produce continuous spasm because of absence of interneurons in the brainstem. ${ }^{7}$ D

The exact mechanism leading to spinal myoclonus is so far unknown. In the literature a number of explanations have been given: a reduced number of inhibitory interneurons, facilitating alpha motor neuron discharge; sublethal injury leading to increased $\vec{F}$ excitability; ${ }^{8}$ neuronal destruction in middle and ven- $\overrightarrow{0}$ tral zones of grey matter ${ }^{9}$ and physiological dis- $\frac{C}{0}$ turbance of the gamma-loop efferent system. We believe that the myoclonus in the case presented here $\mathbb{\otimes}$ can be explained by the histological findings in the lumbar segment.

Neoplastic disease as a cause for the neurological $\vec{\circ}$ symptoms seems improbable. At the time of diagnosis CSF cytology was repeatedly negative and further extensive work-up was unrevealing. The muscular rigidity remained present throughout life and at nec- $\frac{7}{0}$ ropsy no tumour involvement of the spinal cord was found. The distinct neoplastic cells, resembling small or cell carcinoma, in the spinal roots were found in the setting of widespread metastatic disease. Both mus- $\infty$ cular rigidity and myoclonus are incompatible with a 을 root lesion. 5

Muscular rigidity alone has been described in intra medullary spinal cord tumours, subacute necrotising myelopathy, birth trauma and intraspinal cat $\overrightarrow{0}$ itation. $^{2310}$ Spinal myoclonus has been more fre quently observed and associated with various abnormalities of the spinal cord. ${ }^{11}$ Both muscular rigidity and spinal myoclonus have been observed in one case of encephalomyelitis of unknown origin. ${ }^{6}$ Myoclonus has been reported in association with paraneoplastic disease..$^{1213}$

Although this case of a combination of segmental rigidity and spinal myoclonus has not been reported before as a remote effect of cancer, the histological findings most resemble those found in paraneoplastic subacute motor neuronopathy. ${ }^{14}$ In this syndrome prominent segmental neuronal degeneration was found to be restricted to the anterior horn and mild posterior column degeneration in patients with malignant lymphoma. These patients developed a subacute lower motor neuron syndrome with a usually benign course. A more severe neurological picture, but with similar histology has been described in Hodgkin's lymphoma. ${ }^{1516}$

We gratefully acknowledge the help and comments of Prof dr GThAM Bots. Mrs Marianne Jekel provided secretarial assistance. 


\section{References}

1 Gelfan S, Tarlov IM. Interneurons and rigidity of spinal origin. J Physiol (Lond) 1959;146:594-617.

2 Penry JK, Hoefnagel D, Noort S van den, Denny-Brown D. Muscle spasm and abnormal postures resulting from damage to interneurons in spinal cord. Arch Neurol (Chicago) 1960;3:500-12.

3 Rushworth G, Lishman WA, Hughes JT, Oppenheimer DR. Intense rigidity of the arms due to isolation of motoneurons by a spinal tumour. $J$ Neurol Neurosurg Psychiatry 1961;24:132-42.

4 O'Connor PJ, Wynn Parry CB, Davies R. Continuous muscle spasm in intramedullary tumours of the neuraxis. J Neurol Neurosurg Psychiatry 1966;29:310-4.

5 Tarlov IM. Rigidity in man due to spinal interneuron loss. Arch Neurol 1967;16:536-43.

6 Whiteley AM, Swash M, Urich H. Progressive encephalomyelitis with rigidity. Its relation to "subacute myoclonic spinal neuronitis" and to the "stiff man syndrome". Brain 1976;99:27-42.

7 Porter R. Synaptic potentials in the hypoglossal motoneurons. J Physiol (Lond) 1965;180:209-24.

8 Shivapour E, Teasdall RT. Spinal myoclonus with vacuolar degeneration of anterior horn cells. Arch Neurol 1980;37:451-3.
9 Luttrell ChN, Bang FK, Luxenberg K. Newcastle disease encephalomyelitis in cats II Physiological studies on rhythmic myoclonus. Arch Neurol Psychiatr 1959;81:285-91.

10 Tarlov IM. Deafferentation to relieve spasticity or rigidity: reasons for failure in some cases of paraplegia. $J$ Neurosurg 1966;25:270-4.

11 Frenken CWGM, Notermans SLH, Korten JJ, Horstink MWIM. Myoclonic disorders of spinal origin. Clin Neurol Neurosurg 1976;79:107-18.

12 Wooten GF, Bennett JP. Isolated brachial myoclonus secondary to paraneoplastic encephalopathy. Abstract. Ann Neurol 1984;16:133.

13 Pardo RR, Jankovic J. Clinical manifestations and pharmacological treatment of segmental myoclonus. Abstract. Ann Neurol 1985;18:135.

14 Schold SC, Cho E-S, Somasundaram M, Posner JB. Subacute motor neuronopathy: a remote effect of lymphoma. Ann Neurol 1979;5:271-87.

15 Rowland LP, Schneck SA. Neuromuscular disorders associated with malignant disease. J Chronic Dis 1963;16:777-95.

16 Walton JN, Tomlinson BE, Pearce GW. Subacute "poliomyelitis" and Hodgkin's disease. J Neurol Sci 1968;6:435-45. 